\title{
Specially designed microstrip patch antenna parameter optimization by artificial neural network
}

\author{
Sangeeta Shekhawat ${ }^{1 *}$, Sudhanshu Singh $^{2}$, Kumar Vaibhav ${ }^{2}$ and Ashok Kajala ${ }^{1}$ \\ ${ }^{1}$ Arya Institute of Technology and Engineering, Jaipur Rajasthan, India. \\ ${ }^{2}$ Amity University Rajasthan, Jaipur, Rajasthan, India \\ *Email: sshekhawat@jpr.amity.edu
}

Microstrip patch antennas are having limitations in terms of bandwidth and efficiency, and all these imposed by the very presence of the dielectric substrate which cannot be replaced by any other. So in this proposed paper an artificial neural network (ANN) model has been developed for optimizing the various parameters of specially designed microstrip patch antenna [1]. The difficulty in designing microstrip patch antennas is due to the involvement of a large number of substantial parameters and their associated optimal values in order to circumvent this problem, an alternative solution is achieved using artificial neural networks.

This proposed paper is designed with co-axial probe fed [3] compact size microstrip patch antenna with the basic parameters $L, \varepsilon_{r}, W, h, f_{o}$ for achieve better bandwidth and directivity with efficient radiation pattern and gain. The software which is being used to model and simulate the proposed microstrip patch antenna was IE3D, that can be used to calculate and plot return loss, radiation pattern, smith chart, VSWR, and various other parameters [2].

Structure of proposed antenna is shown in Figure 1a.and its zoomed picture is also shown in Figure 1b.

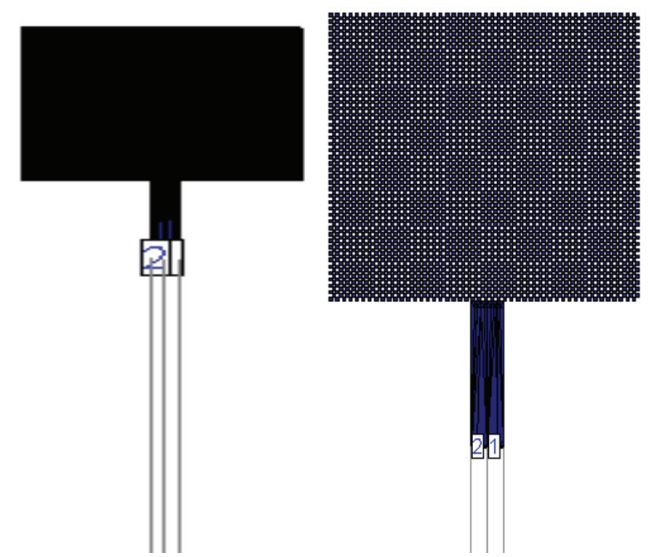

Figure 1: (a) Geometry of proposed structure based microstrip patch antenna (actual structure). (b) Geometry of proposed structure based microstrip patch antenna with dimensions $\quad a=1(\mathrm{~mm}), \quad b=1(\mathrm{~mm}), \quad t=1.524 \quad(\mathrm{~mm})$, permittivity $=4.4$ and grid $\mathrm{size}=.0025 \quad(\mathrm{~mm})$. (zoomed structure)

Result of this proposed antenna has been simulated using IE3D by Zeland software. Figure 2 shows the variation of return loss with frequency. The plot of result shows resonant frequency $.56 \mathrm{GHz}$ and total available impedance band width of $380 \mathrm{MHz}$ that is $13.4 \%$ from the proposed antenna. Minimum -31.98 $\mathrm{db}$ return loss is available at resonant frequency which is significant.

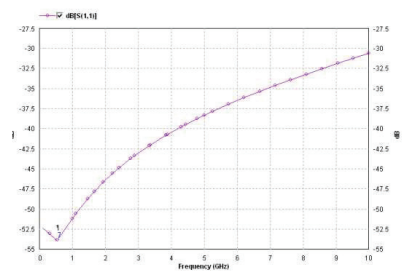

Figure 2: Return losses vs. Frequency curve for proposed antenna

Input impedance curve passing near to the unit impedance circle shows the perfect matching of input. Figure 3 shows the VSWR of the proposed antenna that is $1: 1.01$ at the resonant frequency $.56 \mathrm{GHz}$.

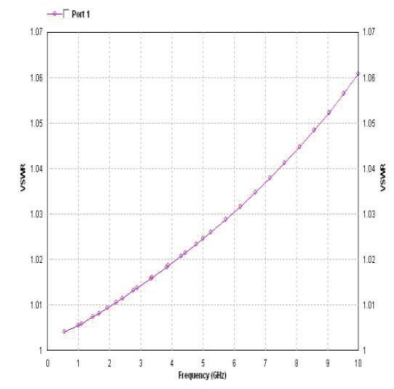

Figure 3: VSWR curve for proposed antenna

The application of artificial neural network ensures an optimum design methodology for microstrip antenna design which is revealed when comparing the results with analytical methods and the results of the simulation software.

\section{References}

1. R.Malmathanraj, S.Thamarai Selvi, Ubiquitous Computing and Communication Journal, 2010, Volume 3 Number 2

2. Jagtar Singh, A.P. Singh, and T.S. Kamal, Proceedings of the World Congress on Engineering 2011 Vol II WCE 2011, July 6 - 8, 2011, London, U.K.

3. C. Borja, G. Font, S. Blanch and J. Romeu, Electronics Letters 2000 Vol. 36 No. 9. 